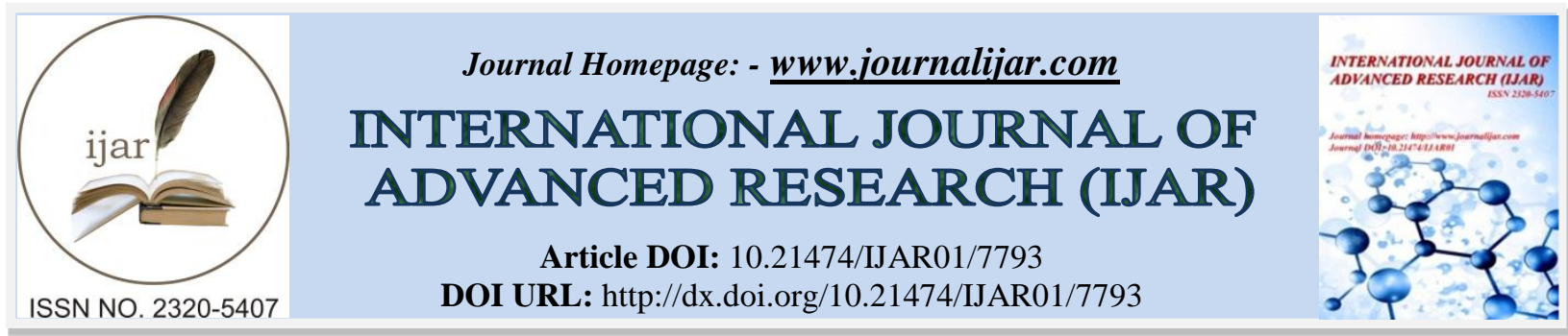

RESEARCH ARTICLE

\title{
UNCOMMON SPLENIC METASTASIS OF AN OVARIAN CYSTADENOCARCINOMA: CASE REPORT AND REVIEW OF THE LITERATURE.
}

\author{
Chaoui Badr, Othman Ayouche, Habib Bellamlih, Nazik Allali, Latifa Chat and Rachida Dafiri. \\ Rabat Children's hospital, Mohamed V University, Rabat, Morocco.
}

\section{Manuscript Info}

\section{Manuscript History}

Received: 2 August 2018

Final Accepted: 4 September 2018

Published: October 2018

Keywords:

Splenic Metastasis; Ovarian Cystadenocarcinoma; Carcinomatosis, Ct.

\begin{abstract}
Introduction: Splenic tumors are rare and often a source of misdiagnosis. The differential diagnosis of splenic metastasis includes benign and malignant etiologies.

Visceral metastases in patients with ovarian cancer represent hematogenous spread of the disease[1].As capsular involvement is more common[2]. Cystic splenic metastasis are even more unusual. Splenectomies for ovarian cancer are usually performed for cytoreduction.

Case Report: We present a rare case of splenic cystic metastasis from ovarian cystadenocarcinoma treated with surgery with neoadjuvant chemotherapy. The evolution was characterized by the death of the patient 06 months after her surgery from a pulmonary embolism.

Conclusion: In summary, splenic metastasis is a relatively rare event. Often asymptomatic, it is usually detected as part of a work up exams of primary cancers, among them ovarian cancer which is highly associated with secondary splenic spread. The work up include imaging studies such as ultrasound and CT scan as well as measurement of CA125 , which often correlated to prognosis. Splenectomy is the treatment of choice. However, it is not done, as splenic metastasis is indicative of advanced disease. In case of a primary ovarian cancer it is often associated to peritoneal carcinomatosis. This case highlights the importance of intraprenchymal cystic splenic metastasis in case of ovarian cystadenocarcinoma as part of the wide range of splenic masses.
\end{abstract}

Copy Right, IJAR, 2018,. All rights reserved.

\section{Introduction:-}

Splenic tumors are rare and often a source of misdiagnosis. The differential diagnosis of splenic metastasis includes benign and malignant etiologies.

Visceral metastases in patients with ovarian cancer represent hematogenous spread of the disease[1].As capsular involvement is more common[2]. Cystic splenic metastasis are even more unusual. Splenectomies for ovarian cancer are usually performed for cytoreduction. We present a rare case of splenic cystic metastasis from ovarian cystadenocarcinoma treated with surgery with neoadjuvant chemotherapy. The evolution was characterized by the death of the patient 06 months after her surgery from a pulmonary embolism. 


\section{Case Report}

A 50 year-old female, with no history of chronic disease, consulted for an abdominal discomfort without functional digestive signs.

Physical examination demonstrated the presence of a large median abdominal mass, which measured $15 \mathrm{~cm}$.

The examination found a painless non pulsatile abdominal mass, with no associated cutaneous signs or vascular nor digestive or urinary complications.

Doppler ultrasound showed large cystic multilocular tumors, the wall of the loculi were regular, thickened (2-5 mm) and hypervascular on Doppler and their contents were are variable with contiguous loculi of different echogenicity (Figure 1).

CT angiography revealed the presence of a Bulky multilocular cystic abdominopelvic mass composed of multiple thickened-walled loculi which increase after injection, of fluid density, some of which have septa as well as intralesional vegetations. It pushes up the digestive loops without invading them. It measures $111 \times 169 \times 247 \mathrm{~mm}$ with bilateral ovarian infiltration(Figure 2). A normal-sized homogeneous spleen with multiple rounded cystic masses of regular contours, fluid density with no enhancement after injection of contrast medium, the largest of which had a liquid-liquid level measuring $57 \times 51 \mathrm{~mm}$.Intraperitoneal effusion of great abundance with peritoneal nodule at the left angle measuring $16 \times 15 \mathrm{~mm}$. As well as a right pleural effusion of average abundance predominant on the right lung. The patient then underwent a same time splenectomy, total abdominal hysterectomy (TAH), bilateral salpingectomy, and omentectomy. After the cytoreductive surgery, the patient underwent a chemotherapy regime.

Pathology from our case revealed ovarian mucinous cystadenocarcinoma poorly differentiated metastatic in the spleen lesions and After surgery the patient's status was regular, with no vascular or digestive complications noted during our monthly follow-up, which consisted of a physical exam, and a US screening as well as a CA-125 dosage.

\section{Discussion:-}

Metastatic carcinoma to the spleen is rare[2,3]. Its incidence has been reported to vary from 9\% to 16\%[4].There appears to be no difference in incidence between men and women, but it is more frequently found in the elderly [5].

The most primary solid cancers associated with splenic spread include of the ovary, colon lung, breast, skin(melanoma), and stomach[6, 7].

In most cases, the spleen is involved as part of a diffuse carcinomatosis, and splenic metastasis reflects diffuse dissemination[3].

Multiple theories have been advanced explaining the physiopathology of splenic spread of metastatic disease [2, 8 , 9] :

1. The paucity of splenic afferent lymphatics

2. The organ motility might explain why it is a rare site of metastasis.

3. the sharp angle of the splenic artery.

4. the rhythmic contractile nature of the spleen.

5. Antitumor activity of the splenic lymphoid tissues.

Clinically, We distinguish 2 types of splenic metastasis [1,2]:

1. Asymptomatic, that are discovered insidiously. Often associated with capsule rupture.

2. Symptomatic lesions tend to be larger, solitary rather than multiple, easy to detect

Ultrasound is the first imaging modality preconized for detecting splenic metastasis; typical findings are of a solitary or multiple well-defined hypoechoic masses

Hypoechoic lesion with a rapid wash-in and washout contrast-enhanced US (CEUS). The cystic appearance is exceptional; it is attributed to a tumoral necrosis of the mass with a hematic content.

On contrast enhanced CT computed, the lesions are usually hypo attenuating with inhomogeneous enhancement after contrast administration, cystic components may be present but are exceptional. 
As for magnetic resonance imaging, the lesions routinely are hyper intense (if melanin or blood containing) on T1WI/T2WI sequences, with a variable enhancement depending on the primary malignancy.

We did not opt for an MRI because we had no characterization problems. We did a contrast enhanced CT in order to evaluate for extension.

Two attitudes have been preconized for histological diagnosis: Splenectomy for prevention of eventual splenic rupture, or direct splenic biopsy.

However, In our case biopsy may not be preconized based on the likely widespread nature of the tumor, the high associated mortality rate, and the poor rentability of biopsy on cystic metastasis[10].In most of reports, the spleen was involved as part of diffuse carcinomatosis, and there are only a few cases of isolated parenchymal metastases [3, 11]. Although splenic metastasis are not included as a criterium in the FIGO classification, Nicklin et al have suggested that splenic parenchymal metastasis is indicative of FIGO stage IV disease.[12].They also suggested that splenic metastasis receive the same consideration as hepatic metastasis.

In our case, although there were peritoneal seeding, the spleen capsule was intact. However we observed a particularity as there were multiple large cystic metastasis who reminisce us of certain parts of the primary which was composed of hematic cystic components. So we concluded, there was no direct invasion. The mechanism would be more in favor of a vascular spread of the disease. She also had widespread visceral, peritoneal, and right diaphragmatic implants, which comfort this theory.

Laparoscopic splenectomy, is preferred over open splenectomy in these cases As it has the advantage of short operating and a quicker recovery time. Also It is associated with less post-operative complications[13].

It has been proposed that patients can begin their chemotherapy sooner after laparoscopic splenectomy than those with an open splenectomy [13,14]. As several authors have suggested that splenectomy may have a role in cytoreduction $[12,15]$. Parenchymal lesions are significantly less likely than surface lesions to respond to chemotherapy. Also, those at relapse were less likely to respond [1].These findings may have implications for further therapeutic evaluation and planification as well as the prevention of further complications.

\section{Conclusion:-}

In summary, splenic metastasis is a relatively rare event. Often asymptomatic, it is usually detected as part of a work up exams of primary cancers, among them ovarian cancer which is highly associated with secondary splenic spread.

The work up include imaging studies such as ultrasound and CT scan as well as measurement of CA-125, which often correlated to prognosis.

Splenectomy is the treatment of choice. However, it is not done, as splenic metastasis is indicative of advanced disease. In case of a primary ovarian cancer it is often associated to peritoneal carcinomatosis. This case highlights the importance of intraprenchymal cystic splenic metastasis in case of ovarian cystadenocarcinoma as part of the wide range of splenic masses.

\section{Conflict Of Interest}

The corresponding author is the guarantor of submission.

\section{Author's Contributions}

Badr Chaoui - Acquisition of data, Drafting the article, Revising it critically for important intellectual content, Final approval of the version to be published

Ayouche Othman - Acquisition of data, Drafting the article, Revising it critically for important intellectual content, Final approval of the version to be published

Habib Bellamlih - Acquisition of data, Drafting the article, Revising it critically for important intellectual content, Final approval of the version to be published

Nazik Allali - Acquisition of data, Drafting the article, Revising it critically for important intellectual content, Final approval of the version to be published

Latifa Chat - Acquisition of data, Drafting the article, Revising it critically for important intellectual content, Final approval of the version to be published

Rachida Dafiri - Acquisition of data, Drafting the article, Revising it critically for important intellectual content, Final approval of the version to be published 


\section{Source Of Support}

None

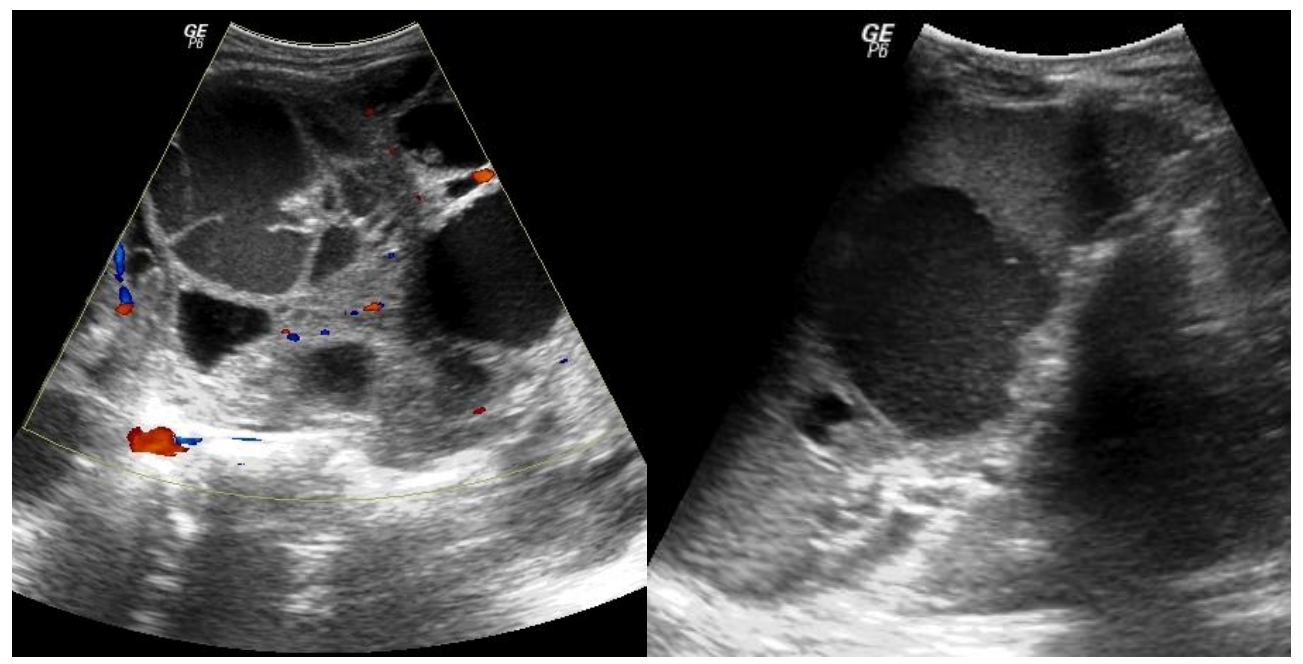

Figure 1:-Abdominal US : a multiloculated cystic pelvic mass with septal vascularization at Doppler exam. Cystic splenic cystic mass with hematic content.

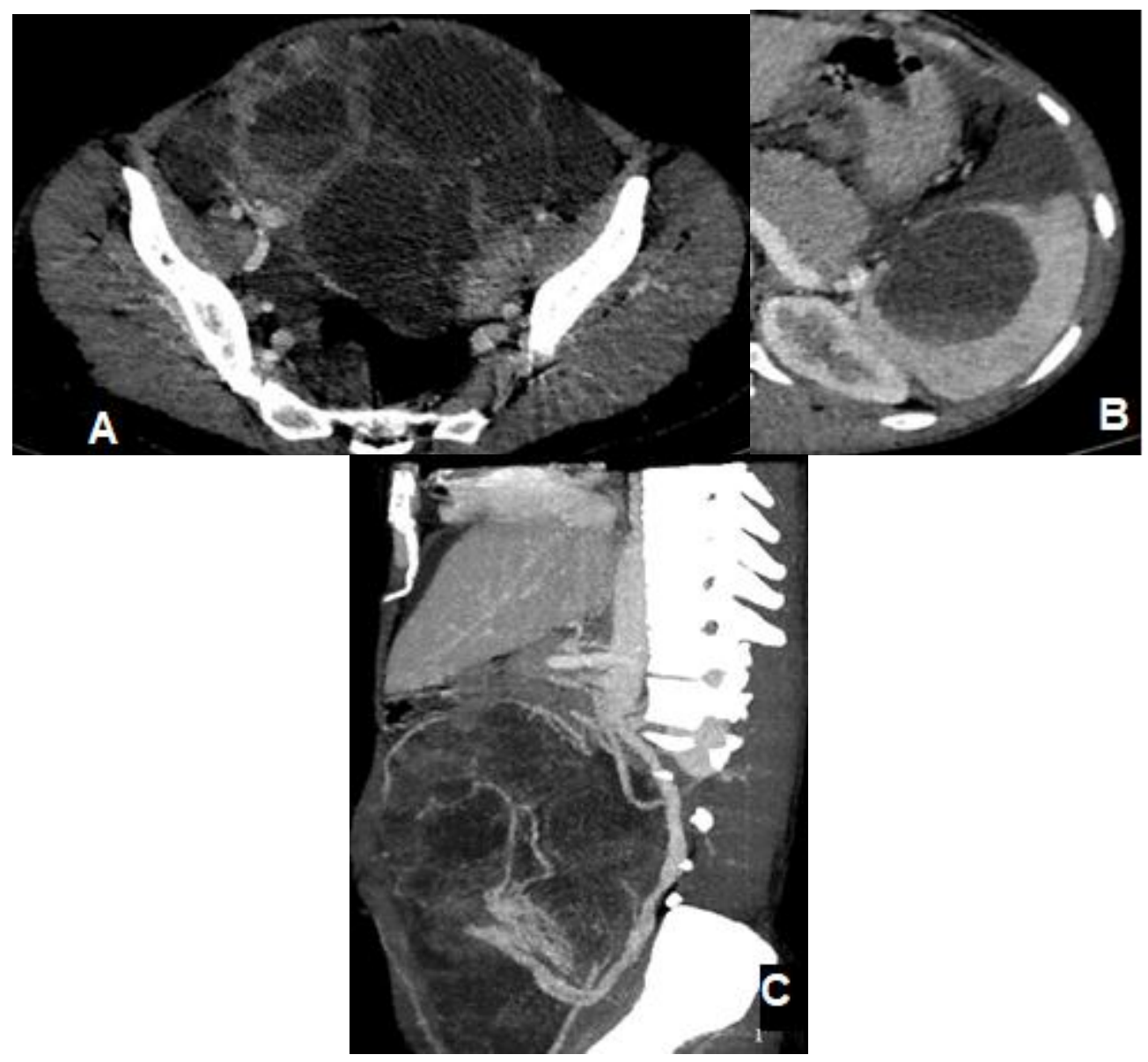

Figure 2:-Contrast enhanced abdominal CT with sagittal MIP section: Two multiloculated latero-uterine formation of which some cysts have liquid-liquid levels(A). On the left mass, there are tortuous irregular vessels on the periphery, which are confluent to the left ovary vein on the sagittal section in $\operatorname{MIP}(\mathrm{C})$. We also identify the splenic cystic metastasis with liquid-liquid level that remind us of the primary tumor(B). 


\section{References:-}

1. Spencer N, Spencer J, Perren T, Lane G. CT appearances and prognostic significance of splenic metastasis in ovarian cancer. Clinical radiology. 1998;53(6):417-21.

2. Ghani AA, Hashmi ZA, Chase DM, Patel SB, Jones DF. Intraparenchymal metastases to the spleen from ovarian cancer: a case report. Journal of medical case reports. 2010;4(1):30.

3. Farias-Eisner R, Braly P, Berek JS. Solitary recurrent metastasis of epithelial ovarian cancer in the spleen. Gynecologic oncology. 1993;48(3):338-41.

4. Abrams HL, Spiro R, Goldstein N. Metastases in carcinoma. Analysis of 1000 autopsied cases. Cancer. 1950;3(1):74-85.

5. Lam K, Tang V. Metastatic tumors to the spleen: a 25-year clinicopathologic study. Archives of pathology \& laboratory medicine. 2000;124(4):526-30.

6. Morgenstern L, Rosenberg J, Geller SA. Tumors of the spleen. World journal of surgery. 1985;9(3):468-76.

7. Compérat E, Bardier-Dupas A, Camparo P, Capron F, Charlotte F. Splenic metastases: clinicopathologic presentation, differential diagnosis, and pathogenesis. Archives of pathology \& laboratory medicine. 2007;131(6):965-9.

8. Warren S, Davis AH. Studies on tumor metastasis: V. The metastases of carcinoma to the spleen. The American Journal of Cancer. 1934;21(3):517-33.

9. Karni D, Kopelman D, Hatoum OA. Solitary splenic metastasis of ovarian carcinoma: a case report. Journal of medical case reports. 2014;8(1):154.

10. Alici S, Kosem M, Kotan C. Isolated splenic metastases occurring as an unknown primary lesion. Journal of postgraduate medicine. 2003;49(1):83.

11. Glezerman M, Yanai-Inbar I, Charuzi I, Katz M, Glasner M, Piura B. Involvement of the spleen in ovarian adenosquamous carcinoma. Gynecologic oncology. 1988;30(1):143-8.

12. Nicklin J, Copeland L, O'Toole R, Lewandowski G, Vaccarello L, Havenar L. Splenectomy as part of cytoreductive surgery for ovarian carcinoma. Gynecologic oncology. 1995;58(2):244-7.

13. Otrock Z, Seoud M, Khalifeh M, Makarem J, Shamseddine A. Laparoscopic splenectomy for isolated parenchymal splenic metastasis of ovarian cancer. International Journal of Gynecological Cancer. 2006;16(5):1933-5.

14. Koh YS, Kim JC, Cho CK. Splenectomy for solitary splenic metastasis of ovarian cancer. BMC cancer. 2004;4(1):96.

15. Sonnendecker EW, Guidozzi F, Margolius KA, Path F. Splenectomy during primary maximal cytoreductive surgery for epithelial ovarian cancer. Gynecologic oncology. 1989;35(3):301-6. 